\title{
Antibacterial and Solubility Optimization of Thiomuracin A
}

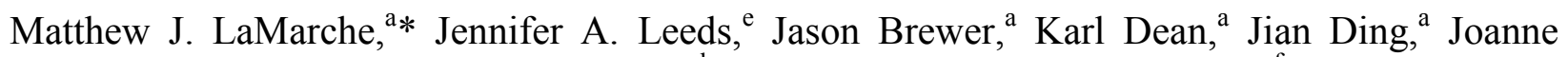
Dzink-Fox, ${ }^{\mathrm{e}}$ Gabe Gamber, ${ }^{\mathrm{a}}$ Akash Jain, ${ }^{\mathrm{d}}$ Ryan Kerrigan, ${ }^{\mathrm{a}}$ Philipp Krastel, ${ }^{\mathrm{f}}$ Kwangho Lee, ${ }^{\mathrm{a}}$ Franco Lombardo, ${ }^{c}$ David McKenney, Georg Neckermann, ${ }^{\mathrm{e}}$ Colin Osborne, ${ }^{\mathrm{e}}$ Deborah Palestrant, ${ }^{\mathrm{b}}$ Michael A. Patane, ${ }^{\mathrm{a}}$ Elin M. Rann, ${ }^{\mathrm{a}}$ Zachary Robinson, ${ }^{\mathrm{a}}$ Esther Schmitt, ${ }^{\mathrm{f}}$ Travis Stams, ${ }^{\mathrm{b}}$ Stacey Tiamfook, ${ }^{\mathrm{e}}$ Donghui Yu, ${ }^{\mathrm{e}}$ Lewis Whitehead ${ }^{\mathrm{a}}$

${ }^{a}$ Global Discovery Chemistry, ${ }^{b}$ Protein Structure Group, ${ }^{c}$ Metabolism and Pharmacokinetics, Novartis Institutes for Biomedical Research, ${ }^{d}$ Chemical and Pharmaceutical Profiling, Novartis Pharmaceuticals, Cambridge, MA 02139, United States. ${ }^{e}$ Infectious Disease Area, Novartis Institutes for Biomedical Research, Emeryville, CA 94608, United States. ${ }^{f}$ Natural Products Unit, Novartis Institutes for Biomedical Research, Basel, Switzerland.

\section{Supporting Information:}

analytical methods, experimental procedures 
Abbreviations:

HATU

i-PrOH

DCM

DMF

TBTU

DCC

$\mathrm{HCl}$

TLC

$\mathrm{MeOH}$

$\mathrm{AcOH}$

TFA

TEA

$\mathrm{Eq}$

$\mathrm{aq}$

h

hep

EtOAc

$10 \% \mathrm{Pd} / \mathrm{C}$
1-[bis(dimethylamino)methylene]-1H-1,2,3-triazolo[4,5-

b]pyridinium 3-oxid hexafluorophosphate)

isopropanol

dichloromethane

dimethylformamide

2-(1H-benzotriazole-1-yl)-1,1,3,3-tetramethyluronium

tetrafluoroborate

$N, N^{\prime}$-Dicyclohexylcarbodiimide

hydrochloric acid

thin layer chromatography

methanol

acetic acid

trifluoroacetic acid

triethylamine

equivalents

aqueous

hours

heptane

ethyl acetate

palladium on carbon; 10 wt. \% loading (dry basis), matrix 
activated carbon, wet support, Degussa type E101 NE/W

inh. inhibition

EtOH ethanol

LC liquid chromatography

LC/MS liquid chromatography mass spectrum

Min minutes

RT room temperature

$\mathrm{R}_{\mathrm{t}} \quad$ retention time

sat. $\quad$ saturated

$\mathrm{m} / \mathrm{z} \quad$ mass to charge MS mass spectrum

HRMS high resolution mass spectrum

$\mathrm{MHz}$ megahertz, $10^{6} \mathrm{~Hz}$

\section{ANALYTICAL METHODS}

NMR: proton spectra are recorded on a Bruker $400 \mathrm{MHz}$ ultrashield spectrometer. Chemical shifts are reported relative to dimethyl sulfoxide $(\delta 2.50)$

LC/MS: Method 1: compounds are analyzed on an Inertsil ODS-3 column (C18, 50 x $4.6 \mathrm{~mm}, 3$ $\mu \mathrm{m})$ with a 2 min gradient elution $\left(20-80 \%\right.$ acetonitrile $/ \mathrm{H}_{2} \mathrm{O} / 5 \mathrm{mM}$ ammonium formate) and a flow rate of $4 \mathrm{ml} / \mathrm{min}$. Method 2: Compounds are analyzed on an Inertsil ODS-3 column (C8, $30 \mathrm{~mm} \times 3.0 \mathrm{~mm}, 3.0 \mu \mathrm{M})$ with a 2 min gradient elution $\left(5-90 \%\right.$ acetonitrile $/ \mathrm{H}_{2} 0 / 5 \mathrm{mM}$ ammonium formate) and a flow rate of $2 \mathrm{ml} / \mathrm{min}$. Method 3: Compounds are analyzed on an 
Inertsil ODS-3 column $(\mathrm{C} 8-3,3.0 \mathrm{~cm} \times 33 \mathrm{~mm} \times 3.0 \mu \mathrm{m})$ with a 2 min gradient elution $(5-95 \%$ acetonitrile/ $\mathrm{H}_{2} 0 / 0.1 \%$ Formic Acid) and flow rate of $2 \mathrm{ml} / \mathrm{min}$.

HPLC purification utilizes a C8 or C18 column $(30 \times 100 \mathrm{~mm}, 5 \mu \mathrm{m}$, brand: Sunfire or XTerra) and is performed with an appropriate gradient according to two methods (unless otherwise noted). Method 1 consists of $0.1 \%$ TFA in 5\%-95\% ACN in $\mathrm{H}_{2} \mathrm{O}$. Method 2 consists of $10 \mathrm{mM} \mathrm{NH}_{4} \mathrm{OH}$ in $5 \%-95 \% \mathrm{ACN}$ in $\mathrm{H}_{2} \mathrm{O}$.

The below compounds (e.g. 1, 2,9) were previously described: J. Med. Chem. 2012, 55, 6934-6941 and WO2008/148754.

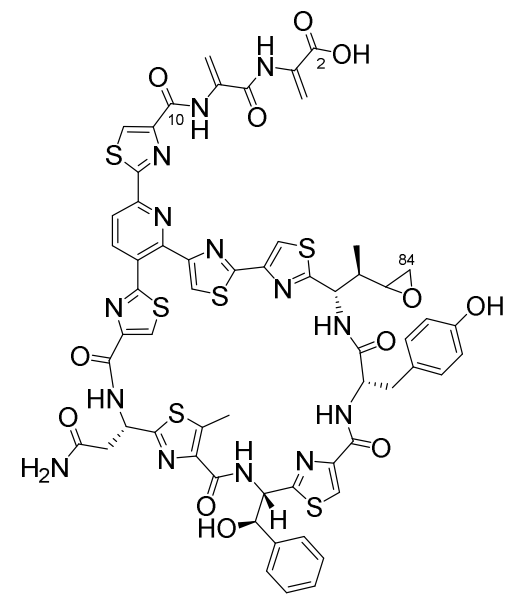

Thiomuracin (1)

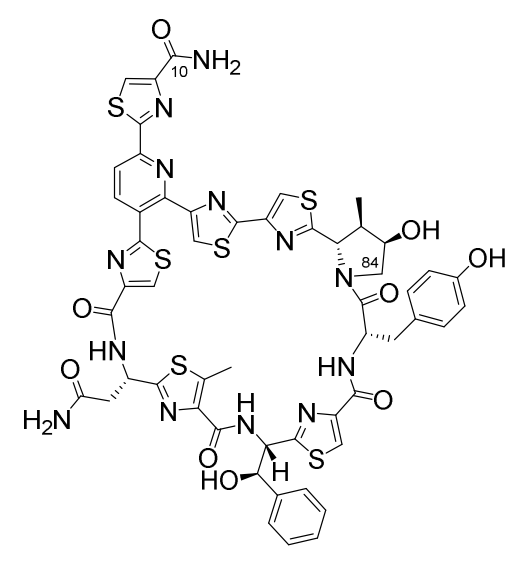

Lead (2)

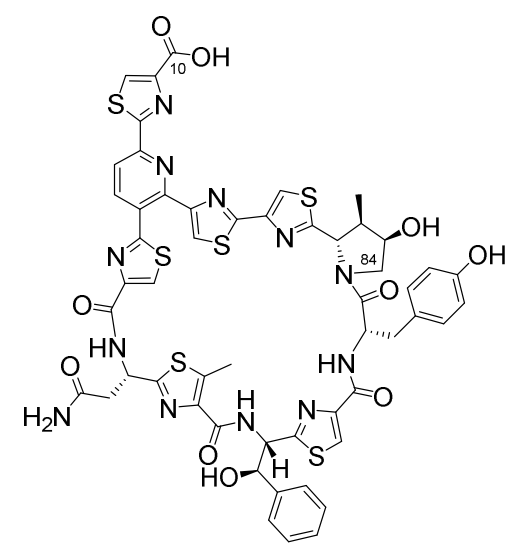

Acid (9)

Preparation of $(1 r, 4 r)-4-\left(\left(\left(2-\left(\left(1^{2} S, 1^{3} R, 1^{4} R, 2^{2} Z, 3^{2} Z, 5^{2} Z, 9^{2} Z, 13^{2} Z, 8 S, 12 S, 16 S\right)-8-(2-a m i n o-2-\right.\right.\right.\right.$ oxoethyl)-1 ${ }^{4}$-hydroxy-12-((R)-hydroxy(phenyl)methyl)-16-(4-hydroxybenzyl)-1 ${ }^{3}, 9^{5}$ dimethyl-6,10,14,17-tetraoxo-7,11,15-triaza-2,3,5,9,13(2,4)-pentathiazola-4(2,3)-pyridina1(2,1)-pyrrolidinacycloheptadecaphane- $4^{6}$-yl)thiazol-4-yl)carbamoyl)oxy)cyclohexane-1carboxylic acid (16): 


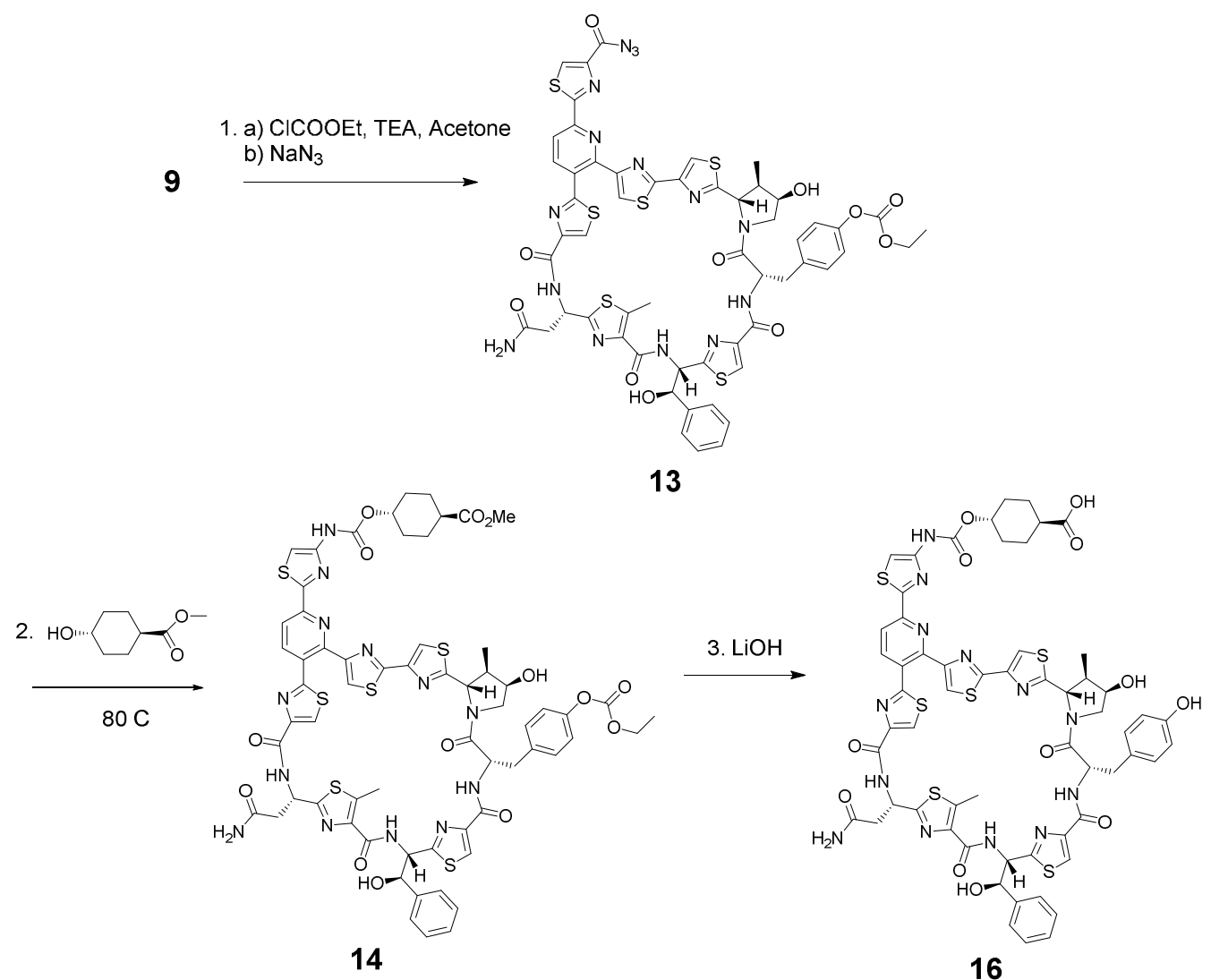

Step 1: To a suspension of the acid (9) (392 $\mathrm{mg}, 0.326 \mathrm{mmol})$ in 11:1 acetone/water $(80.5 \mathrm{~mL})$ was added triethylamine $(0.45 \mathrm{~mL}, 3.26 \mathrm{mmol}, 10 \mathrm{eq})$ and ethyl chloroformate $(0.37 \mathrm{~mL}, 3.91$ mmol, 12 eq). The reaction was stirred at ambient temperature for 1.5 hours. Sodium azide $(0.318 \mathrm{~g}, 4.89 \mathrm{mmol}, 15 \mathrm{eq})$ was added and the reaction was stirred at room temperature until complete. Solvents were evaporated at $20{ }^{\circ} \mathrm{C}$. Then water was added to the residue to precipitate the azide. The azide was suspended three times in water then once in dichloromethane and isolated by filtration to afford azide 13, (241 $\mathrm{mg}, 0.185 \mathrm{mmol}, 57 \%$ yield) LC/MS: $[\mathrm{M}+\mathrm{H}]^{+} 1298, \mathrm{R}_{\mathrm{t}}=1.47 \min .(\operatorname{method} 2)$.

Step 2: The azide (13) (241 $\mathrm{mg}, 0.185 \mathrm{mmol}$ ) was combined with 4-hydroxycyclohexanecarboxylic acid methyl ester $(88 \mathrm{mg}, 0.556 \mathrm{mmol}, 3.0 \mathrm{eq})$ and oven dried 3 angstrom molecular sieves $(241 \mathrm{mg})$ in 1,4-dioxane $(6 \mathrm{~mL})$ and stirred at $80{ }^{\circ} \mathrm{C}$ for $7 \mathrm{~h}$. Solvent was 
evaporated and the residue was purified by silica gel chromatography (gradient elution: 3-100\% $\mathrm{MeOH} / \mathrm{DCM})$ to afford ester 14, (100 mg, $0.700 \mathrm{mmol}, 38 \%$ yield) LC/MS: $[\mathrm{M}+2 \mathrm{H}]^{+} 1429, \mathrm{R}_{\mathrm{t}}=$ $1.53 \min (\operatorname{method} 2)$.

Step 3: The ester (14) (100 mg, $0.700 \mathrm{mmol})$ was dissolved in 1:1 dichloromethane/methanol (6 mL). $4 \mathrm{M}$ aqueous lithium hydroxide solution $(0.3 \mathrm{~mL}, 1.2 \mathrm{mmol}, 1.7 \mathrm{eq})$ was added at ambient temperature with stirring. After 9.5 hours, glacial acetic acid was added $(0.5 \mathrm{~mL})$ and the solvent was evaporated. The residue is purified by HPLC ( 20 to $100 \%$ acetonitrile in water $+0.1 \%$ TFA) to afford compound (16), $\left(28.2 \mathrm{mg}, 0.070 \mathrm{mmol}, 30 \%\right.$ yield). LC/MS: $[\mathrm{M}+\mathrm{H}]^{+} 1342, \mathrm{R}_{\mathrm{t}}=1.26$ $\min \left(\operatorname{method~2).~}{ }^{1} \mathrm{H}\right.$ NMR (400 MHz, DMSO- $\left.d_{6}\right) \delta$ ppm 12.13 (s, $\left.1 \mathrm{H}\right), 10.67$ (s, $\left.1 \mathrm{H}\right), 9.16$ (s, 1 H), $8.77(\mathrm{~d}, J=7.7 \mathrm{~Hz}, 1 \mathrm{H}), 8.58(\mathrm{~s}, 1 \mathrm{H}), 8.47-8.38(\mathrm{~m}, 2 \mathrm{H}), 8.21(\mathrm{~d}, J=4.8 \mathrm{~Hz}, 2 \mathrm{H}), 8.14$ $(\mathrm{d}, J=8.1 \mathrm{~Hz}, 1 \mathrm{H}), 7.62(\mathrm{~d}, J=9.3 \mathrm{~Hz}, 1 \mathrm{H}), 7.51(\mathrm{~s}, 1 \mathrm{H}), 7.38(\mathrm{~s}, 1 \mathrm{H}), 7.27-7.17(\mathrm{~m}, 4 \mathrm{H})$, $7.08-7.00$ (m, $2 \mathrm{H}), 6.92(\mathrm{~d}, J=8.4 \mathrm{~Hz}, 2 \mathrm{H}), 6.72-6.57(\mathrm{~m}, 3 \mathrm{H}), 6.53(\mathrm{~s}, 1 \mathrm{H}), 6.34$ (d, $J=3.7$ $\mathrm{Hz}, 1 \mathrm{H}), 5.49-5.42(\mathrm{~m}, 1 \mathrm{H}), 5.34-5.26(\mathrm{~m}, 2 \mathrm{H}), 5.19-5.10(\mathrm{~m}, 1 \mathrm{H}), 5.07-4.97(\mathrm{~m}, 1 \mathrm{H})$, $4.73-4.57$ (m, $2 \mathrm{H}), 4.17$ (s, $1 \mathrm{H}), 3.93-3.73(\mathrm{~m}, 2 \mathrm{H}), 2.99-2.90(\mathrm{~m}, 1 \mathrm{H}), 2.79-2.69(\mathrm{~m}, 1$ H), $2.66(\mathrm{~s}, 3 \mathrm{H}), 2.30-2.14(\mathrm{~m}, 2 \mathrm{H}), 2.06-1.84(\mathrm{~m}, 5 \mathrm{H}), 1.53-1.39(\mathrm{~m}, 4 \mathrm{H}), 1.04(\mathrm{~d}, J=$ $6.7 \mathrm{~Hz}, 3 \mathrm{H}) .{ }^{13} \mathrm{C}$ NMR (101 MHz, DMSO) $\delta \mathrm{ppm} 176.15,173.81,171.66,170.45,168.51$, $165.75,164.68,163.86,161.44,160.28,160.18,158.81,155.97,153.54,153.18,150.48,150.19$, $149.86,149.34,147.28,147.05,141.53,140.87,140.05,140.01,130.32,127.62,127.37,127.26$ $126.60,126.44,126.29,125.61,122.57,117.82,115.47,115.18,103.44,72.68,72.03,71.07$, $64.43,57.40,56.19,51.61,48.69,47.03,40.99,37.47,37.22,30.42,26.49,12.06,10.50$. HRMS: $\mathrm{C}_{60} \mathrm{H}_{56} \mathrm{~N}_{13} \mathrm{O}_{12} \mathrm{~S}_{6}, 1342.2500,(\mathrm{M}+\mathrm{H})^{+}$, calc'd =1342.2496.

Preparation of (1r,4r)-4-(((2-((1 $\left.{ }^{2} S, 1^{3} R, 1^{4} R, 2^{2} Z, 3^{2} Z, 5^{2} Z, 9^{2} Z, 13^{2} Z, 8 S, 12 S, 16 S\right)-8-(2-a m i n o-2-$ oxoethyl)-1 ${ }^{4}$-hydroxy-12-((R)-hydroxy(phenyl)methyl)-16-(4-hydroxybenzyl)-1 ${ }^{3}, 9^{5}$ - 
dimethyl-6,10,14,17-tetraoxo-7,11,15-triaza-2,3,5,9,13(2,4)-pentathiazola-4(2,3)-pyridina-

\section{1(2,1)-pyrrolidinacycloheptadecaphane- ${ }^{6}$-yl)thiazol-4-yl)(4-}

carboxybutyl)carbamoyl)oxy)cyclohexane-1-carboxylic acid (3):

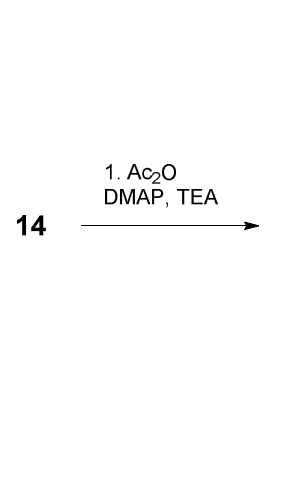

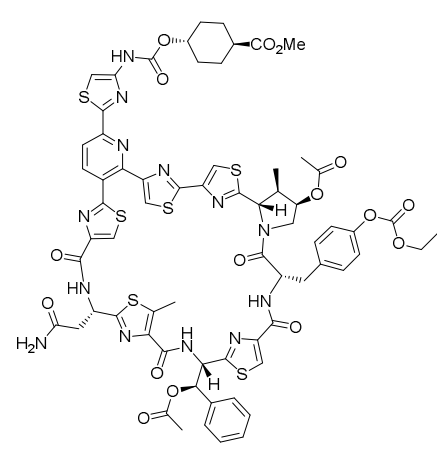

15

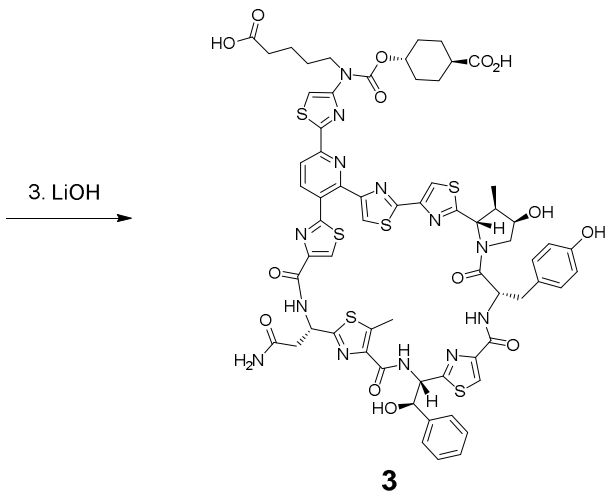

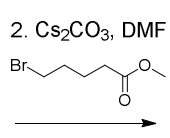

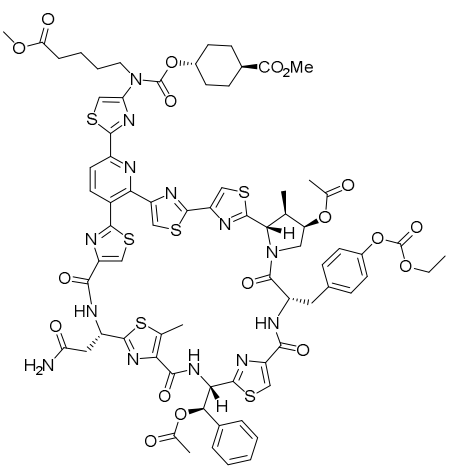

17

Step 1: To a solution of ester (14) $(680 \mathrm{mg}, 0.476 \mathrm{mmol})$ in dichloromethane $(57 \mathrm{~mL}, 8.35 \mathrm{mM})$ was added triethylamine $(0.6 \mathrm{~mL}, 4.3 \mathrm{mmol}, 9.0 \mathrm{eq})$, acetic anhydride $(0.44 \mathrm{~mL}, 4.66 \mathrm{mmol}, 9.8$ eq) and 4-di(methylamino)pyridine $(57 \mathrm{mg}, 0.467 \mathrm{mmol}, 0.98 \mathrm{eq})$ at $20{ }^{\circ} \mathrm{C}$. The reaction was stirred at room temperature for 1 hour. The solvents were evaporated and the residue was purified by silica gel chromatography (gradient elution $0-10 \%$ i-PrOH/DCM) to afford diacetate (15), (600 mg, $0.397 \mathrm{mmol}, 83 \%$ yield). LC/MS: $\left[\mathrm{M}+\mathrm{H}_{3} \mathrm{O}\right]^{+} 1531, \mathrm{R}_{\mathrm{t}}=1.65 \mathrm{~min}(\operatorname{method} 2)$.

Step 2: To a solution of the diacetate (15) $(600 \mathrm{mg}, 0.397 \mathrm{mmol})$ in $18 \mathrm{~mL} \mathrm{N,N-}$ dimethylformamide (18 mL, $0.02 \mathrm{M})$ was added oven dried 4 angstrom molecular sieves (1.15 g), 
methyl-5-bromovalerate $(1.13 \mathrm{~mL}, 7.88 \mathrm{mmol}, 20 \mathrm{eq})$ and cesium carbonate $(1.29 \mathrm{~g}, 3.96 \mathrm{mmol}$, $10 \mathrm{eq})$. The reaction was stirred at room temperature until complete. Acetic acid $(5 \mathrm{~mL})$ and silica (6 g) were added and the mixture was evaporated to dryness. The residue was purified by silica gel chromatography to afford diester (17), (522 mg, $0.310 \mathrm{mmol}, 78 \%$ yield). LC/MS: $[\mathrm{M}+\mathrm{H}]^{+} 1627, \mathrm{R}_{\mathrm{t}}=1.74 \min (\operatorname{method} 1)$.

Step 3: Diester (17) (505 mg, $0.310 \mathrm{mmol})$ was dissolved in 1:1 dichloromethane/methanol (30 $\mathrm{mL}, 10.4 \mathrm{mM})$ and chilled to $0{ }^{\circ} \mathrm{C}$. Saturated lithium hydroxide solution $(5 \mathrm{~mL})$ was added slowly and the reaction was stirred at $0{ }^{\circ} \mathrm{C}$ until complete. Acetic acid $(10 \mathrm{~mL})$ was added and the solvent was evaporated. The residue purified by HPLC to afford diacid (3), (240 mg, 0.166 mmol, 54\% yield). LC/MS: $[\mathrm{M}+2 \mathrm{H}]^{+} 1443, \mathrm{R}_{\mathrm{t}}=1.39 \min (\operatorname{method} 3) .{ }^{1} \mathrm{H}$ NMR (400 MHz, DMSO- $\left.d_{6}\right) \delta$ ppm $8.78(\mathrm{~d}, J=7.8 \mathrm{~Hz}, 1 \mathrm{H}), 8.58(\mathrm{~s}, 1 \mathrm{H}), 8.45(\mathrm{~d}, J=6.5 \mathrm{~Hz}, 1 \mathrm{H}), 8.42-8.37$ (m, $1 \mathrm{H}), 8.27-8.17(\mathrm{~m}, 3 \mathrm{H}), 7.70(\mathrm{~s}, 1 \mathrm{H}), 7.61(\mathrm{~d}, J=9.2 \mathrm{~Hz}, 1 \mathrm{H}), 7.37(\mathrm{~s}, 1 \mathrm{H}), 7.28-7.16$ (m, 4 H), $7.08-6.98$ (m, 2 H), $6.96-6.89$ (m, 2 H), 6.67 (s, 1 H), 6.61 (d, J=8.4 Hz, 2 H), 6.35 (s, $1 \mathrm{H}), 5.50-5.41(\mathrm{~m}, 1 \mathrm{H}), 5.30(\mathrm{~d}, J=4.6 \mathrm{~Hz}, 2 \mathrm{H}), 5.20-5.10(\mathrm{~m}, 1 \mathrm{H}), 5.10-4.98(\mathrm{~m}, 1$ H), $4.74-4.60$ (m, 2 H), $4.23-4.12(\mathrm{~m}, 1 \mathrm{H}), 4.07-3.92$ (m, 2 H), $3.92-3.74(\mathrm{~m}, 2 \mathrm{H}), 3.00-$ $2.91(\mathrm{~m}, 1 \mathrm{H}), 2.79-2.69(\mathrm{~m}, 1 \mathrm{H}), 2.66(\mathrm{~s}, 3 \mathrm{H}), 2.59-2.52(\mathrm{~m}, 1 \mathrm{H}), 2.32-2.13(\mathrm{~m}, 4 \mathrm{H})$, $2.07-1.82(\mathrm{~m}, 5 \mathrm{H}), 1.78-1.36(\mathrm{~m}, 9 \mathrm{H}), 1.04(\mathrm{~d}, J=6.7 \mathrm{~Hz}, 3 \mathrm{H}) .{ }^{13} \mathrm{C}$ NMR $(101 \mathrm{MHz}$, DMSO) $\delta$ ppm 176.14, 174.38, 173.81, 171.71, 170.46, 168.54, 165.63, 164.63, 163.17, 161.43, $160.18,158.80,155.97,153.55,153.36,151.11,150.39,150.07,149.32,147.28,147.02,141.51$, $140.82,140.00,130.31,127.60,127.34,126.45,126.24,125.63,122.58,118.00,73.58,71.95$, $71.08,64.45,57.40,56.20,51.57,48.68,47.04,41.69,41.45,40.81,37.45,37.21,33.21,30.74$ $30.03,27.67,27.02,26.61,26.16,21.63,19.92,12.06,10.49$. HRMS: $\mathrm{C}_{65} \mathrm{H}_{64} \mathrm{~N}_{13} \mathrm{O}_{14} \mathrm{~S}_{6}$, 1442.3033, $(\mathrm{M}+\mathrm{H})^{+}$, calc'd $=1442.3020$. 
Preparation of 5-(2-((1 $\left.{ }^{2} S, 1^{3} R, 1^{4} R, 2^{2} Z, 3^{2} Z, 5^{2} Z, 9^{2} Z, 13^{2} Z, 8 S, 12 S, 16 S\right)-8$-(2-amino-2-oxoethyl)$1^{4}$-hydroxy-12-((R)-hydroxy(phenyl)methyl)-16-(4-hydroxybenzyl)-1 ${ }^{3}, 9^{5}$-dimethyl6,10,14,17-tetraoxo-7,11,15-triaza-2,3,5,9,13(2,4)-pentathiazola-4(2,3)-pyridina-1(2,1)pyrrolidinacycloheptadecaphane- $4^{6}$-yl)thiazole-4-carboxamido)pentanoic acid (10):
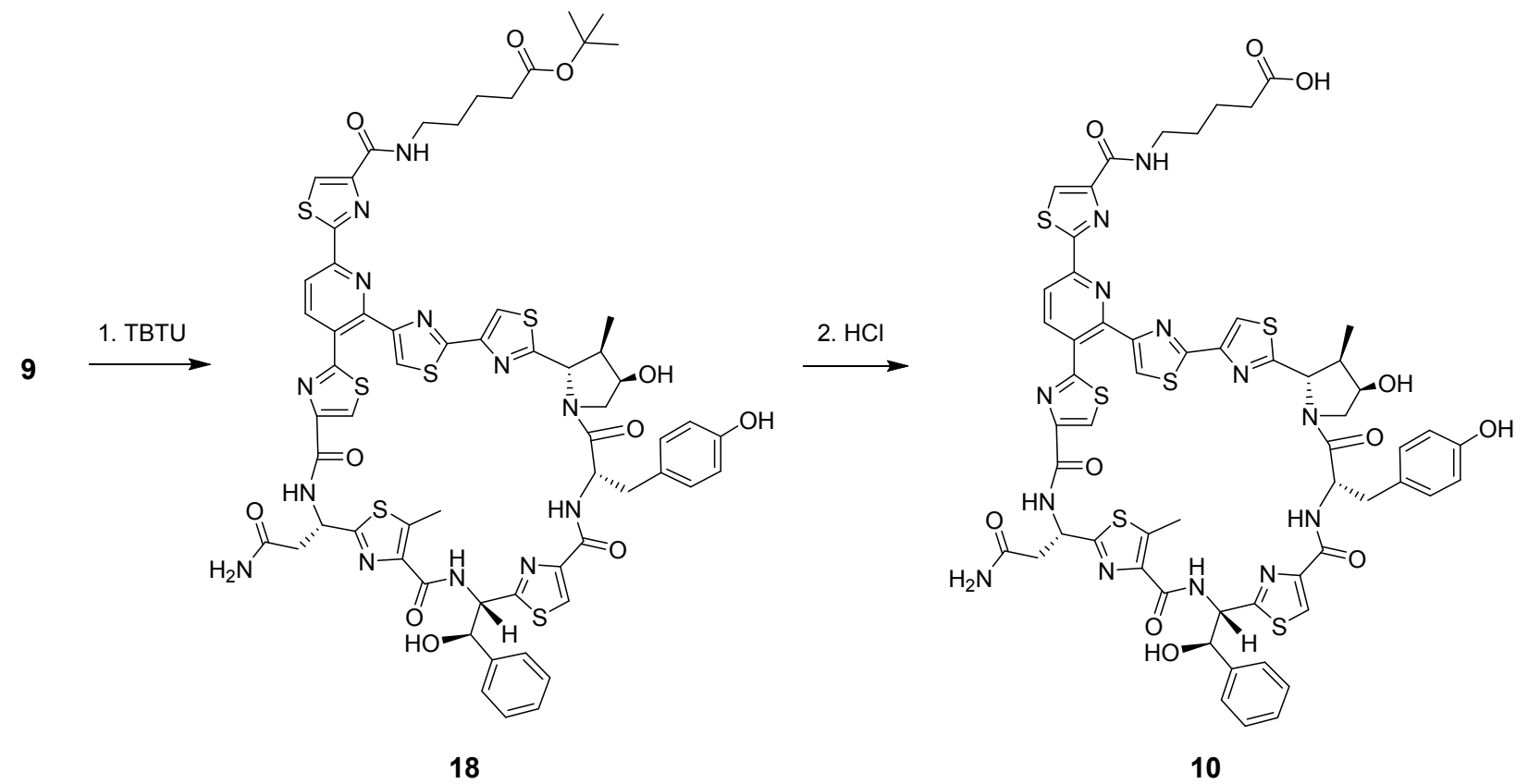

Step 1: To a solution of acid (9) $(500 \mathrm{mg}, 0.416 \mathrm{mmol})$ in DCM $(25 \mathrm{~mL})$ and DMF $(10 \mathrm{~mL})$ was added pyridine ( $2 \mathrm{~mL}, 24.7 \mathrm{mmol}, 60 \mathrm{eq}$ ) followed by tert-butyl 5 -aminopentanoate ( $300 \mathrm{mg}$, $1.73 \mathrm{mmol}, 4.2 \mathrm{eq})$ in DCM (3 mL). Then TBTU (300 mg, $0.931 \mathrm{mmol}, 2.2 \mathrm{eq})$ was added. The mixture was stirred at ambient temperauter for $18 \mathrm{~h}$. Silica was added and the mixture was evaporated to dryness. The residue was purified by silica gel chromatography to afford the $t$ butyl ester (18), (565 mg, $0.416 \mathrm{mmol},>100 \%$ yield).

Step 2: To a solution of $t$-butyl ester $(\mathbf{1 8})(565 \mathrm{mg}, 0.416 \mathrm{mmol})$ in 1,4-dioxane $(10 \mathrm{~mL})$ was added $4 \mathrm{M} \mathrm{HCl}$ in 1,4-dioxane $(25 \mathrm{~mL}, 100 \mathrm{mmol}, 1 \mathrm{eq})$. The resulting suspension was stirred at ambient temperature for $2 \mathrm{~h}$ then evaporated to dryness. The residue was purified by HPLC to 
afford mono-acid (10: $242 \mathrm{mg}, 0.177 \mathrm{mmol}, 43 \%$ yield). LC/MS: $[\mathrm{M}+\mathrm{H}]^{+}$1300, $\mathrm{R}_{\mathrm{t}}=1.23 \mathrm{~min}$ (method 1). ${ }^{1} \mathrm{H}$ NMR (400 MHz, DMSO- $\left.d_{6}\right) \delta$ ppm 9.18 (br. s., $\left.1 \mathrm{H}\right), 8.81(\mathrm{~d}, J=7.53 \mathrm{~Hz}, 1 \mathrm{H}$ ), $8.73(\mathrm{t}, J=5.77 \mathrm{~Hz}, 1 \mathrm{H}), 8.61(\mathrm{~s}, 1 \mathrm{H}), 8.37-8.56(\mathrm{~m}, 4 \mathrm{H}), 8.26(\mathrm{~s}, 1 \mathrm{H}), 8.22(\mathrm{~s}, 1 \mathrm{H}), 7.63(\mathrm{~d}$, $J=9.04 \mathrm{~Hz}, 1 \mathrm{H}), 7.37$ (s, $1 \mathrm{H}), 7.13$ - 7.28 (m, $4 \mathrm{H}), 6.98$ - 7.11 (m, $2 \mathrm{H}), 6.84$ - 6.99 (m, $2 \mathrm{H})$, 6.67 - $6.76(\mathrm{~m}, 1 \mathrm{H}), 6.54$ - $6.66(\mathrm{~m}, 2 \mathrm{H}), 6.26$ - $6.46(\mathrm{~m}, 1 \mathrm{H}), 5.38$ - $5.52(\mathrm{~m}, 1 \mathrm{H}), 5.24$ - 5.37 (m, 2 H), 5.09 - $5.21(\mathrm{~m}, 1 \mathrm{H}), 4.91-5.09(\mathrm{~m}, 1 \mathrm{H}), 4.69(\mathrm{~d}, J=10.04 \mathrm{~Hz}, 1 \mathrm{H}), 4.08-4.30(\mathrm{~m}, 1$ H), 3.72 - $3.95(\mathrm{~m}, 2 \mathrm{H}), 3.23$ - $3.37(\mathrm{~m}, 2 \mathrm{H}), 2.69$ - $3.02(\mathrm{~m}, 2 \mathrm{H}), 2.66(\mathrm{~s}, 3 \mathrm{H}), 2.55$ - $2.58(\mathrm{~m}$, $1 \mathrm{H}), 2.24-2.37$ (m, $2 \mathrm{H}), 1.84-2.24$ (m, $2 \mathrm{H}), 1.41-1.70$ (m, $4 \mathrm{H}), 1.04$ (d, J=6.53 Hz, $3 \mathrm{H})$. ${ }^{13} \mathrm{C}$ NMR (101 MHz, DMSO) $\delta$ ppm 174.37, 173.72, 171.59, 170.34, 168.40, 166.80, 165.69, $164.53,161.37,160.24,160.17,160.11,158.74,155.89,153.35,151.45,150.31,150.15,149.27$ $147.19,146.98,141.45,140.75,139.98,139.94,130.24,127.67,127.55,127.29,126.67,126.36$, $126.22,125.52,122.66,118.64,115.40,115.11,71.96,71.00,64.33,57.33,56.10,51.54,48.59$, $46.93,38.29,37.37,37.13,33.21,28.72,21.86,11.98$, 10.42. HRMS: $\mathrm{C}_{58} \mathrm{H}_{54} \mathrm{~N}_{13} \mathrm{O}_{11} \mathrm{~S}_{6}$, 1300.2386, $(\mathrm{M}+\mathrm{H})^{+}$, calc'd $=1300.2390$.

Preparation of $(1 r, 4 r)-4-\left(2-\left(\left(1^{2} S, 1^{3} R, 1^{4} R, 2^{2} Z, 3^{2} Z, 5^{2} Z, 9^{2} Z, 13^{2} Z, 8 S, 12 S, 16 S\right)-8\right.\right.$-(2-amino-2oxoethyl)-1 ${ }^{4}$-hydroxy-12-((R)-hydroxy(phenyl)methyl)-16-(4-hydroxybenzyl)-1 ${ }^{3}, 9^{5}$ dimethyl-6,10,14,17-tetraoxo-7,11,15-triaza-2,3,5,9,13(2,4)-pentathiazola-4(2,3)-pyridina1(2,1)-pyrrolidinacycloheptadecaphane-4 ${ }^{6}$-yl)thiazole-4-carboxamido)cyclohexane-1carboxylic acid (11): 

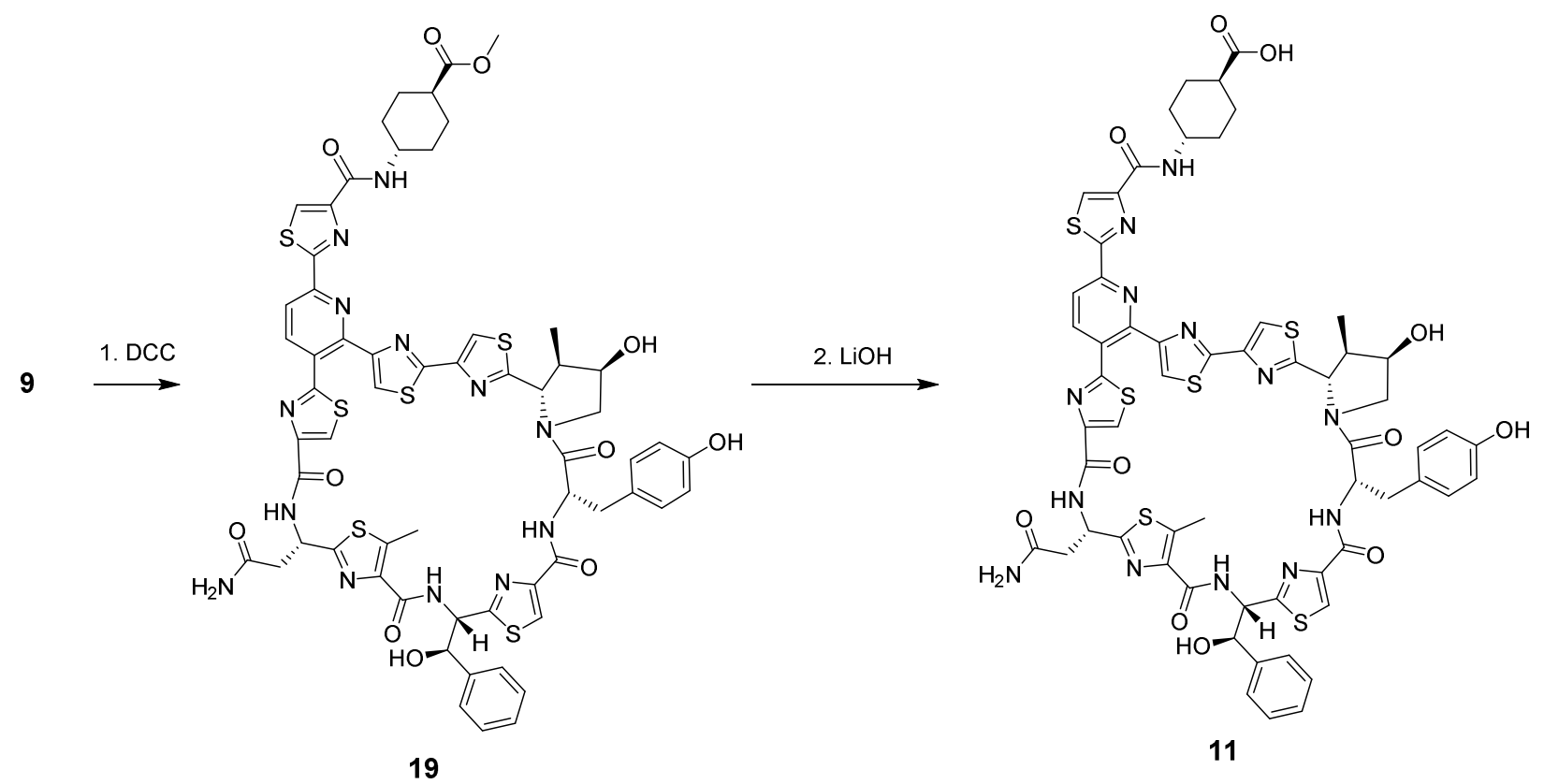

Step 1: To a solution of acid (9: $70 \mathrm{mg}, 0.058 \mathrm{mmol})$ in DCM $(10 \mathrm{~mL}, 5.83 \mathrm{mM})$ was added $N$, $N$ '-dicyclohexylcarbodiimide immobilized on polystyrene $[1.9 \mathrm{mmol} / \mathrm{g}](92 \mathrm{mg}, 0.175 \mathrm{mmol}, 3$ eq), trans-4-amino-cyclohexylcarboxylic acid methyl esther hydrochloride (14 mg, $0.087 \mathrm{mmol}$, $1.5 \mathrm{eq})$, and pyridine $(30 \mu \mathrm{L})$. The reaction was stirred for $12 \mathrm{~h}$ and adsorbed onto silica. The residue was purified by silica gel chromatography (gradient elution: $0-10 \% \mathrm{MeOH} / \mathrm{DCM}$ ) to afford ester 19 (25 mg, 0.019 mmol, 32\% yield). LC/MS: $[\mathrm{M}+\mathrm{H}]^{+} 1340, \mathrm{R}_{\mathrm{t}}=1.43 \mathrm{~min}(\operatorname{method}$ 1). ${ }^{1} \mathrm{H}$ NMR (DMSO-d6) $\delta$ ppm $9.14(\mathrm{~s}, 1 \mathrm{H}), 8.78(\mathrm{~d}, 1 \mathrm{H}), 8.59(\mathrm{~s}, 1 \mathrm{H}), 8.48-8.41(\mathrm{~m}, 4 \mathrm{H})$, $8.34(\mathrm{~d}, 1 \mathrm{H}), 8.24(\mathrm{~s}, 1 \mathrm{H}), 8.20(\mathrm{~s}, 1 \mathrm{H}), 7.61(\mathrm{~d}, 1 \mathrm{H}), 7.36(\mathrm{~s}, 1 \mathrm{H}), 7.24-7.19(\mathrm{~m}, 4 \mathrm{H}), 7.04-$ 7.02 (m, 2 H), 6.92 (d, 2 H), 6.66-6.63 (br. s, 1 H), 6.60 (d, 2 H), 6.32 (d, 1 H), 5.45-5.42 (dd, 1 H), 5.31-5.27 (m, 2 H), 5.18-5.12 (m, $1 \mathrm{H})$, 5.04-4.97 (m, $1 \mathrm{H}), 4.68(\mathrm{~d}, 1 \mathrm{H}), 4.18-4.14(\mathrm{~m}, 1 \mathrm{H})$, 3.89-3.74 (m, $3 \mathrm{H}), 3.61(\mathrm{~s}, 3 \mathrm{H}), 2.97-2.91(\mathrm{~m}, 1 \mathrm{H}), 2.75-2.70(\mathrm{~m}, 1 \mathrm{H}), 2.66-2.65(\mathrm{~m}, 1 \mathrm{H})$, $2.65(\mathrm{~s}, 3 \mathrm{H}), 2.57-2.54(\mathrm{~m}, 1 \mathrm{H}), 2.34-2.26(\mathrm{~m}, 1 \mathrm{H}), 2.21-2.15(\mathrm{~m}, 1 \mathrm{H}), 2.01-1.85(\mathrm{~m}, 5 \mathrm{H})$, 1.58-1.41 (m, $4 \mathrm{H}), 1.03(\mathrm{~d}, 3 \mathrm{H})$. HRMS: $\mathrm{C}_{61} \mathrm{H}_{58} \mathrm{~N}_{13} \mathrm{O}_{11} \mathrm{~S}_{6}, 1340.2687,(\mathrm{M}+\mathrm{H})^{+}$, calc'd = 1340.2703 . 
Step 2: To a solution of the ester intermediate $(25 \mathrm{mg}, 0.019 \mathrm{mmol})$ in $2: 1$ dichloromethane/methanol $(15 \mathrm{~mL}, 1.24 \mathrm{mM})$, saturated lithium hydroxide solution $(5 \mathrm{~mL})$ was added slowly and the reaction was stirred at ambient temperature for 6 hours. The residue was adsorbed onto silica gel and filtered through a silica gel plug $(10 \% \mathrm{MeOH} / \mathrm{DCM}+0.1 \% \mathrm{AcOH})$ then purified via preparatory TLC (10\% MeOH/DCM) to afford affords acid 11 (8 mg, 0.006 mmol, 32\% yield). LC/MS: $[\mathrm{M}+\mathrm{H}]^{+}=1326, \mathrm{R}_{\mathrm{t}}=1.17 \min (\operatorname{method} 1) .{ }^{1} \mathrm{H}$ NMR $\left(\mathrm{DMSO}-d_{6}\right) \delta$ ppm 12.05 (br. s, 1 H), 9.14 (s, 1 H), 8.78 (d, 1 H), 8.59 (s, 1 H), 8.49-8.40 (m, 4 H), 8.33 (d, 1 H), $8.24(\mathrm{~s}, 1 \mathrm{H}), 8.20(\mathrm{~s}, 1 \mathrm{H}), 7.61(\mathrm{~d}, 1 \mathrm{H}), 7.36(\mathrm{~s}, 1 \mathrm{H})$, 7.23-7.20 (m, $4 \mathrm{H})$, 7.04-7.00 (m, 2 H), $6.92(\mathrm{~d}, 2 \mathrm{H}), 6.66-6.63(\mathrm{~m}, 1 \mathrm{H}), 6.60$ (d, $2 \mathrm{H}), 6.32$ (d, $1 \mathrm{H}), 5.44$ (dd, $1 \mathrm{H})$, 5.30-5.28 (m, 2 H), 5.19-5.13 (m, $1 \mathrm{H})$, 5.04-4.97 (m, $1 \mathrm{H}), 4.69$ (d, $1 \mathrm{H}), 4.18-4.14(\mathrm{~m}, 1 \mathrm{H}), 3.89-3.74(\mathrm{~m}, 3 \mathrm{H})$, 2.97-2.91 (m, $1 \mathrm{H}), 2.76-2.68(\mathrm{~m}, 1 \mathrm{H}), 2.65(\mathrm{~s}, 3 \mathrm{H}), 2.53-2.51(\mathrm{~m}, 1 \mathrm{H}), 2.23-2.14(\mathrm{~m}, 2 \mathrm{H})$, 2.01-1.85 (m, $5 \mathrm{H}), 1.57-1.37(\mathrm{~m}, 4 \mathrm{H}), 1.03(\mathrm{~d}, 3 \mathrm{H}) .{ }^{13} \mathrm{C}$ NMR (101 MHz, DMSO) $\delta \mathrm{ppm}$ $176.44,173.81,171.66,170.42,168.46,166.90,165.81,164.63,161.46,160.34,160.20,159.46$, $158.83,158.15,157.85,157.54,157.24,155.98,153.42,151.50,150.37,150.22,149.35,147.27$, $147.07,141.53,140.78,140.08,140.03,130.32,127.79,127.64,127.38,126.95,126.72,126.45$ $126.30,125.59,122.73,118.89,115.84,115.51,115.19,72.06,71.08,64.41,57.42,56.18,54.90$ $51.63,48.65,47.46,46.99,41.74,37.45,37.20,31.21,27.78,12.06,10.50$. HRMS: $\mathrm{C}_{60} \mathrm{H}_{56} \mathrm{~N}_{13} \mathrm{O}_{11} \mathrm{~S}_{6}, 1326.2540,(\mathrm{M}+\mathrm{H})^{+}$, calc'd = 1326.2546.

Preparation of $(1 r, 4 r)-4-\left(2-\left(\left(1^{2} S, 1^{3} R, 1^{4} R, 2^{2} Z, 3^{2} Z, 5^{2} Z, 9^{2} Z, 13^{2} Z, 8 S, 12 S, 16 S\right)-8-(2-a m i n o-2-\right.\right.$ oxoethyl)-1 ${ }^{4}$-hydroxy-12-((R)-hydroxy(phenyl)methyl)-16-(4-hydroxybenzyl)-1 ${ }^{3}, 9^{5}$ dimethyl-6,10,14,17-tetraoxo-7,11,15-triaza-2,3,5,9,13(2,4)-pentathiazola-4(2,3)-pyridina1(2,1)-pyrrolidinacycloheptadecaphane- $4^{6}$-yl)- $N$-(4-carboxybutyl)thiazole-4carboxamido)cyclohexane-1-carboxylic acid (12): 


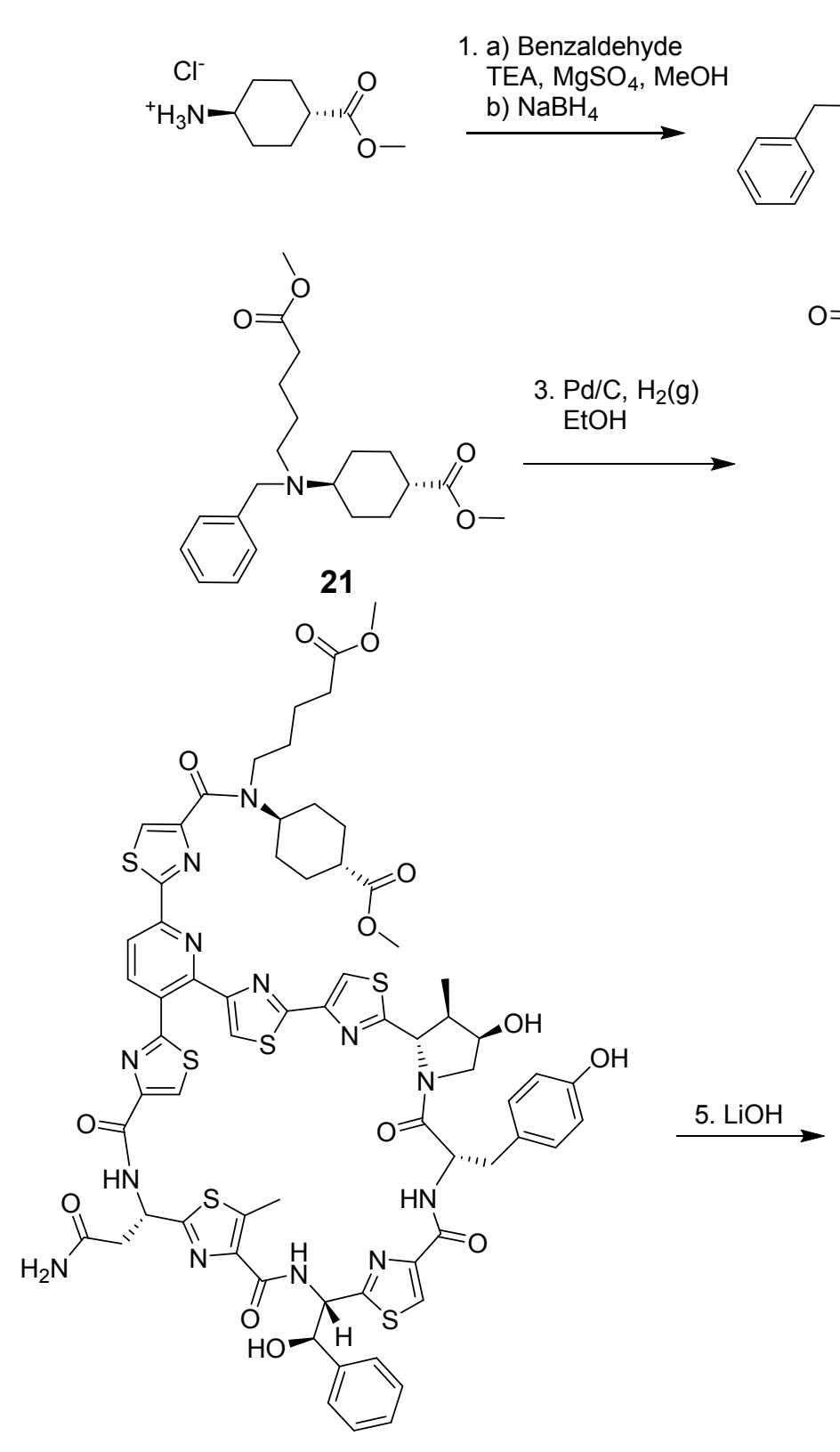

23
2. $\mathrm{Cs}_{2} \mathrm{CO}_{3}$, DMF

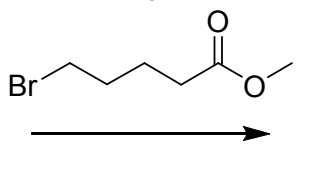

20<smiles>[R20]C1CCC(NCCCCC(=O)OC)CC1</smiles>

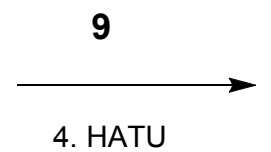

4. HATU

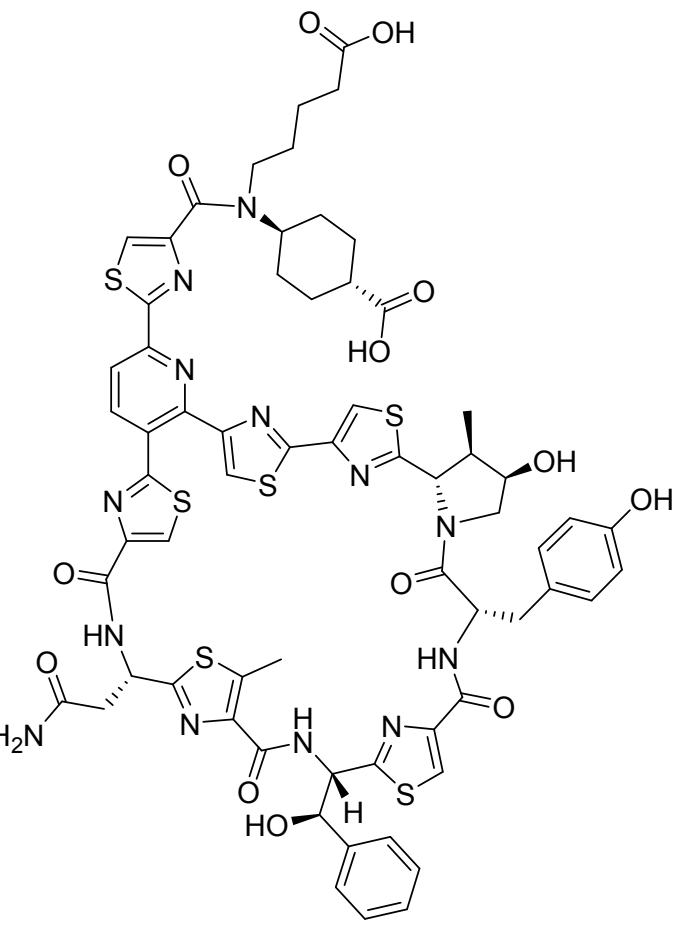

12

Step 1: To a solution of the trans-4-aminocyclohexane carboxylic acid methyl ester (500 mg, $2.58 \mathrm{mmol})$ in $\mathrm{MeOH}(3 \mathrm{~mL})$ was added TEA $(0.14 \mathrm{~mL}, 1.03 \mathrm{mmol}, 0.4 \mathrm{eq}), \mathrm{MgSO}_{4}(466 \mathrm{mg}$, $3.87 \mathrm{mmol}, 1.5 \mathrm{eq})$, and benzaldehyde $(0.29 \mathrm{~mL}, 2.84 \mathrm{mmol}, 1.1 \mathrm{eq})$. The reaction mixture was stirred for 1 hour at $22{ }^{\circ} \mathrm{C}$. The resulting suspension was cooled to $0{ }^{\circ} \mathrm{C}$ and $\mathrm{NaBH}_{4}(586 \mathrm{mg}$, $15.49 \mathrm{mmol}, 6.0 \mathrm{eq})$ was added portionwise. The reaction mixture was stirred for 1 hour at $0{ }^{\circ} \mathrm{C}$. 
Then water $(20 \mathrm{~mL})$ was added and the mixture was stirred for $20 \mathrm{~min}$ at $22{ }^{\circ} \mathrm{C}$ then extracted with EtOAc. The organic layers were combined and dried over $\mathrm{Na}_{2} \mathrm{SO}_{4}$, filtered and concentrated to afford trans-4-benzylaminocyclohexane carboxylic acid methyl ester (20), (535 mg, 2.16 mmol, $84 \%$ yield).

Step 2: To a solution of the trans-4-benzylaminocyclohexane carboxylic acid methyl ester 20 (534 mg, $2.16 \mathrm{mmol})$ in DMF (18 mL, $0.12 \mathrm{M})$ was added $\mathrm{Cs}_{2} \mathrm{CO}_{3}(2.3 \mathrm{~g}, 6.91 \mathrm{mmol}, 3.2 \mathrm{eq})$ and methyl 5-bromovalerate $(1.8 \mathrm{~mL}, 12.96 \mathrm{mmol}, 6.0 \mathrm{eq})$. The reaction mixture was stirred for 18 hours at $22{ }^{\circ} \mathrm{C}$. Then water was added and the mixture was extracted with EtOAc. The organic layers were combined and dried over $\mathrm{Na}_{2} \mathrm{SO}_{4}$, filtered and concentrated. The solid was purified by silica gel chromatography, eluting with EtOAc/Hep (0-60 \%) to afford 4-[benzyl-(4methoxycarbonyl-butyl)-amino]-cyclohexanecarboxylic acid methyl ester 21 (270 mg, 0.747 mmol, $35 \%$ yield).

Step 3: To a solution of the 4-[benzyl-(4-methoxycarbonyl-butyl)-amino]-cyclohexanecarboxylic acid methyl ester 21 (270 mg, $0.747 \mathrm{mmol})$ in EtOH (10 mL, $0.075 \mathrm{M})$ was added 10\% Pd/C (36 $\mathrm{mg}, 0.034 \mathrm{mmol}, 0.05 \mathrm{eq})$. The reaction mixture was stirred for 18 hours at $22{ }^{\circ} \mathrm{C}$ under hydrogen gas at atmospheric pressure. The resulting suspension was then filtered though celite and all solvents are evaporated in vacuo to afford 4-(4-methoxycarbonyl-butylamino)cyclohexanecarboxylic acid methyl ester 22 (203 mg, $0.747 \mathrm{mmol}, 100 \%$ yield).

Step 4: To a suspension of the acid (9) (300 mg, $0.250 \mathrm{mmol}$ ) and amine (22) (75 mg, 0.275 mmol, $1.1 \mathrm{eq})$ in DMF $(10 \mathrm{~mL}, 0.025 \mathrm{M})$ was added $N, N$-diisopropylethylamine $(0.13 \mathrm{~mL}, 0.750$ mmol, $3.0 \mathrm{eq})$ and HATU (143 $\mathrm{mg}, 0.376 \mathrm{mmol}, 1.5 \mathrm{eq})$. The reaction mixture was stirred for 18 
hours, then adsorbed onto silica and purified by silica gel chromatography to afford the diester (23), (210 mg, $0.144 \mathrm{mmol}, 58 \%$ yield).

Step 5: To the diester (23) $(210 \mathrm{mg}, 0.144 \mathrm{mmol})$ in tetrahydrofuran (5 mL, $0.029 \mathrm{M})$ was added saturated lithium hydroxide solution $(50 \mu \mathrm{L})$. The reaction mixture was stirred at ambient temperature for $30 \mathrm{~min}$, quenched with $12 \mathrm{M}$ hydrochloric acid $(50 \mu \mathrm{L})$, and concentrated in vacuo. The residue was purified by HPLC to afford diacid 12 (64 $\mathrm{mg}, 0.045 \mathrm{mmol}, 31 \%$ yield). ${ }^{1} \mathrm{H}$ NMR (400 MHz, DMSO-d 6 ) $\delta$ ppm 9.15 (s, $\left.1 \mathrm{H}\right), 8.79(\mathrm{~d}, J=7.6 \mathrm{~Hz}, 1 \mathrm{H}), 8.69$ (s, $\left.1 \mathrm{H}\right), 8.61$ $-8.58(\mathrm{~m}, 1 \mathrm{H}), 8.49-8.41(\mathrm{~m}, 2 \mathrm{H}), 8.33-8.23(\mathrm{~m}, 2 \mathrm{H}), 8.21(\mathrm{~s}, 1 \mathrm{H}), 7.67-7.57$ (m, $1 \mathrm{H})$, $7.41-7.35$ (m, 1 H), $7.29-7.16$ (m, 5 H), $7.07-6.99$ (m, 2 H), $6.99-6.88$ (m, 2 H), 6.67 (s, 1 H), $6.61(\mathrm{~d}, J=8.4 \mathrm{~Hz}, 2 \mathrm{H}), 6.34(\mathrm{~s}, 1 \mathrm{H}), 5.45(\mathrm{dd}, J=6.4,4.8 \mathrm{~Hz}, 1 \mathrm{H}), 5.30(\mathrm{~d}, J=4.5 \mathrm{~Hz}, 1$ H), $5.20-5.11(\mathrm{~m}, 1 \mathrm{H}), 5.07-4.97(\mathrm{~m}, 1 \mathrm{H}), 4.70(\mathrm{~d}, J=10.3 \mathrm{~Hz}, 1 \mathrm{H}), 4.21-4.14(\mathrm{~m}, 1 \mathrm{H})$, $3.93-3.75(\mathrm{~m}, 3 \mathrm{H}), 3.69-3.21(\mathrm{~m}, 10 \mathrm{H}), 2.99-2.90(\mathrm{~m}, 1 \mathrm{H}), 2.79-2.69(\mathrm{~m}, 1 \mathrm{H}), 2.66(\mathrm{~s}, 3$ H), $2.59-2.52(\mathrm{~m}, 1 \mathrm{H}), 2.25-2.12(\mathrm{~m}, 2 \mathrm{H}), 2.07-1.13(\mathrm{~m}, 6 \mathrm{H}), 1.04(\mathrm{~d}, J=6.7 \mathrm{~Hz}, 4 \mathrm{H})$. ${ }^{13} \mathrm{C}$ NMR (101 MHz, DMSO) $\delta$ ppm 176.28, 174.35, 174.22, 173.76, 171.61, 170.37, 168.45, $167.43,166.21,165.62,164.48,164.42,163.24,161.90,161.36,160.21,160.08,158.73,155.89$, $153.40,153.34,152.22,150.37,150.11,149.28,148.66,147.18,146.96,141.45,140.98,139.93$ $131.66,130.24,127.70,127.54,127.29,126.63,126.36,126.21,125.54,122.67,118.44,115.41$, $115.11,71.92,70.99,64.35,57.32,56.11,51.52,48.62,46.96,37.38,37.15,34.24,33.36,29.81$, 28.46, 27.93, 22.26, 11.98, 10.42. HRMS: $\mathrm{C}_{65} \mathrm{H}_{63} \mathrm{~N}_{13} \mathrm{O}_{13} \mathrm{~S}_{6}, \quad$ 1426.3071, $(\mathrm{M}+\mathrm{H})^{+}$, calc'd $=$ 1426.3071 . 